\title{
Self-Stabilizing Transpiration in Synthetic Leaves
}

\author{
Weiwei Shi, ${ }^{\dagger}$ Joshua R. Vieitez, ${ }^{\dagger}$ Austin S. Berrier, ${ }^{\dagger}$ Matthew W. Roseveare, ${ }^{\dagger}$ Daniel A. Surinach, ${ }^{\dagger}$ \\ Bernadeta R. Srijanto, ${ }^{\ddagger}$ C. Patrick Collier, ${ }^{\ddagger}$ and Jonathan B. Boreyko*, $\dagger, \S$ \\ ${ }^{\dagger}$ Department of Biomedical Engineering and Mechanics and ${ }^{\S}$ Department of Mechanical Engineering, Virginia Tech, Blacksburg, \\ Virginia 24061, United States \\ ${ }^{\ddagger}$ Center for Nanophase Materials Sciences, Oak Ridge National Laboratory, Oak Ridge, Tennessee 37831, United States
}

\section{Supporting Information}

ABSTRACT: Over the past decade, synthetic trees have been engineered to mimic the transpiration cycle of natural plants, but the leaves are prone to dry out beneath a critical relative humidity. Here, we create large-area synthetic leaves whose transpiration process is remarkably stable over a wide range of humidities, even without synthetic stomatal chambers atop the nanopores of the leaf. While the water menisci cannot initially withstand the Kelvin stress of the subsaturated air, they self-stabilized by locally concentrating vapor within the top layers of nanopores that have dried up. Transpiration rates were found to vary nonmonotonically with the ambient humidity because of the tradeoff of dry air increasing the retreat length of the menisci. It is our hope that these findings will encourage the development of large-area synthetic trees that exhibit excellent stability and high throughput for water-harvesting applications.

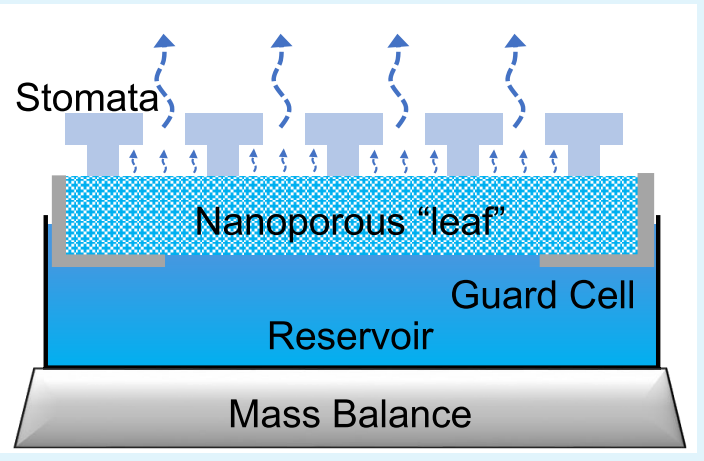

KEYWORDS: synthetic leaf, synthetic tree, nanopores, Laplace pressure, Kelvin pressure

\section{INTRODUCTION}

Transpiration in plants is a process where the ascent of water is powered by a negative water pressure within the leaves, which is generated because of a mismatch in water activity between the saturated leaf and dry air. ${ }^{1-8}$ According to cohesiontension theory, this negative water pressure is transmitted from the leaves all the way down the xylem conduits to the roots, resulting in a hydraulic load that passively pumps water up the tree. $^{9-19}$ Despite its negative pressure, water in trees usually manages to maintain a metastable liquid phase because of the strong cohesive attraction of the water molecules. ${ }^{20-23}$ For over a century, scientists have mimicked natural transpiration using primitive synthetic trees. For example, in 1895 Dixon and Joly attached porous cups to both ends of a tube and pumped water continuously from the bottom cup (i.e., the "reservoir") to the top cup (the "leaf"). ${ }^{24}$

In the past decade, advances in nanofabrication and microfluidic techniques have enabled the fabrication of more sophisticated synthetic trees that promote transpiration across highly controlled architectures. ${ }^{3}$ The earliest example of a modern synthetic tree was demonstrated in 2008, where Wheeler and Stroock pumped water across a microchannel (i.e., "xylem conduit") connecting a pair of nanoporous hydrogels representing the reservoir and a leaf. ${ }^{25}$ Inspired by this work, follow-up reports have also fabricated synthetic trees to characterize the flow of water across a single conduit ${ }^{25-28}$ or across natural wood cuts. ${ }^{29}$ In other cases, synthetic leaves have been directly interfaced with a reservoir (i.e., no intermediate xylem conduits) to focus on characterizing the magnitude and stability of the negative water pressure. $^{20,21,30-33}$ By using increasingly small pore sizes $(\sim 1 \mathrm{~nm}$ diameter) to increase the maximum possible Laplace pressure, these synthetic leaves demonstrated negative pressures as high as $P_{\text {leaf }} \approx-100 \mathrm{MPa}^{34}$ These massive hydraulic loads generated by synthetic leaves are of appeal for passive pumping applications. ${ }^{26,27,35}$ They are also useful for energy harvesting, by injecting bubbles in the flow such that periodic interfaces move along electrodes, ${ }^{36}$ or for thermal management, in the form of evaporative cooling ${ }^{37}$ or heat pipes. ${ }^{38}$ By integrating sensors into a synthetic leaf, it can even be used as a microtensiometer for measuring the water potential of synthetic or natural plants. ${ }^{39,40}$

However, the menisci within synthetic leaves are known to become unstable beneath a critical relative humidity. The magnitude of this critical humidity can be quite high, which poses a severe obstacle for using synthetic trees in real-life environments. For example, a synthetic leaf with pores of radius $r_{\text {pore }} \approx 7 \mathrm{~nm}$ exhibits dry-out for humidities below $85 \% .^{21}$ Even when using the smallest possible nanopores $\left(r_{\text {pore }}\right.$ $\approx 1.5 \mathrm{~nm}$ ), the water menisci become unstable for humidities below $58 \% .^{34}$ The reason for this instability is the mismatch in water activity between the subsaturated air and saturated water

Received: January 3, 2019

Accepted: March 15, 2019

Published: March 26, 2019 

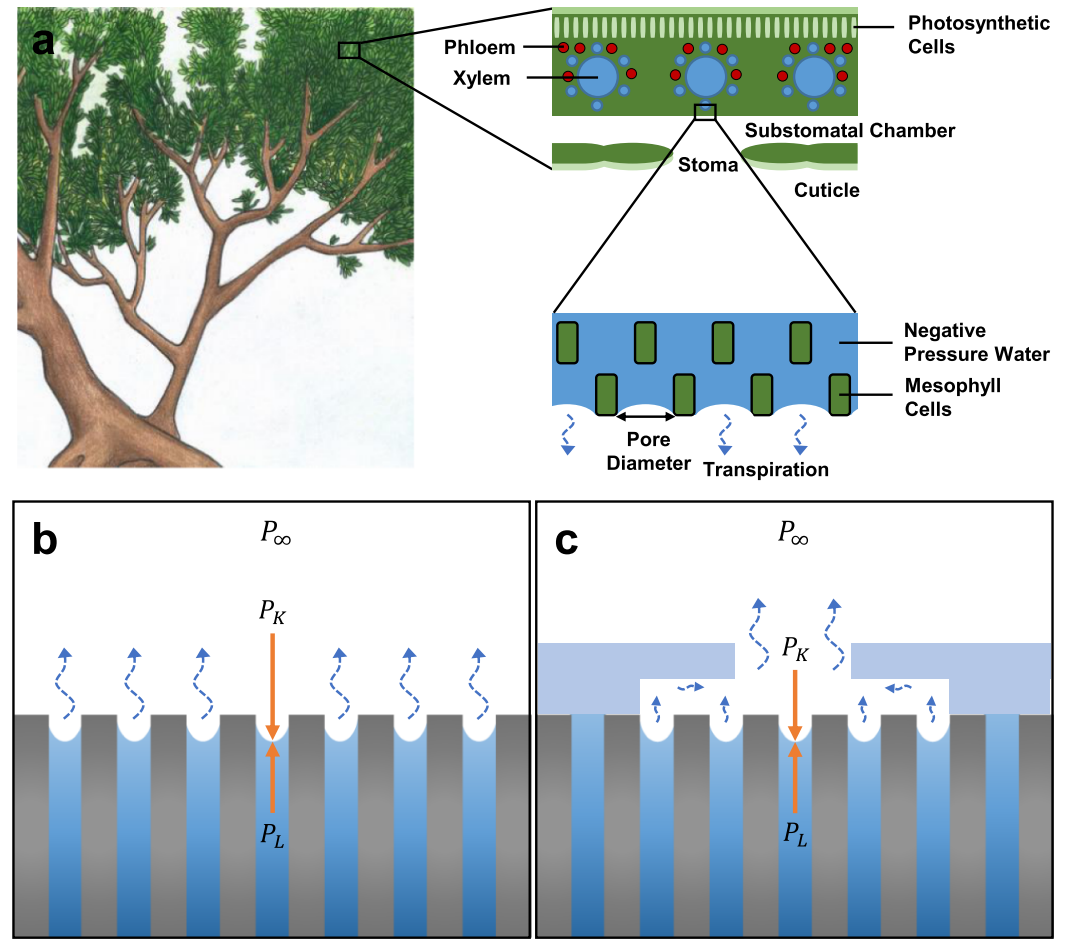

Figure 1. Effects of stomata on transpiration. (a) Illustration of the transpiration cycle of a tree. Water evaporating from a leaf into a subsaturated environment generates a negative pressure, in a manner conceptually analogous to concave water menisci held within nanopores (bottom inset). This water vapor initially concentrates in substomatal cavities and diffuses into the ambient via stomatal apertures (top inset). (b) Without the stomata, the large mismatch in water activity across the interface manifests itself as a Kelvin pressure $\left(P_{\mathrm{K}}\right)$ that outcompetes the negative Laplace pressure of the concave menisci $\left(P_{\mathrm{L}}\right)$. This makes it impossible for the menisci at the outer surface of the nanopores to achieve equilibrium. (c) In the presence of stomatal chambers, the local humidity above the menisci is increased to lessen $P_{\mathrm{K}}$, such that equilibrium is now possible.

across the menisci, which manifests itself as a mechanical pressure (Kelvin pressure). This Kelvin pressure continuously increases with decreasing relative humidity; beneath the critical humidity even the maximum Laplace pressure possible for a given pore size is unable to balance the Kelvin pressure. ${ }^{3,32,34}$ This is problematic for synthetic tree systems because once the interfaces completely recede from the nanopores and enter the adjacent xylem conduits, the nanoscale Laplace pressure producing the suction is lost.

One recent report did show that as the unstable menisci retreat within the nanopores, they can eventually equilibrate as the relative humidity increases within the nanopores. ${ }^{34}$ However, their synthetic leaf was only open to the ambient along the side walls of the thin nanoporous film, such that the menisci retreated along the plane of the film. Such an approach restricts the evaporation of water to a one-dimensional interface, ${ }^{21,32,34}$ which is not practical for real-life applications where a high water throughput across a two-dimensional area is desirable. In contrast, the transpiration of natural leaves occurs across its entire face, and is remarkably stable given its exposure to a wide range of humidities. The primary difference between most synthetic leaves and natural leaves is that the former lack stomata, which are ubiquitous to the latter (save for scant exceptions where synthetic stomata were deployed $^{26,27}$ ). Stomata comprise substomatal chambers that trap the vapor emanating from the underlying nanopores, bleeding it out through a stomatal aperture (Figure 1a). ${ }^{41-44}$ We hypothesize that the remarkable stability of natural leaves can be attributed to their stomata, which serve to concentrate the local humidity directly above the water menisci (Figure $1 b, c)$.
Here, the throughput and stability of transpiration across synthetic leaves are characterized with stomata optionally overlaid atop the nanopores. Across a wide range of ambient humidities, we found that water menisci within the nanopores were self-stabilizing both with and without the stomata attached. When saying that transpiration is "self-stabilizing," we mean that the local humidity directly above the menisci becomes higher than that in the ambient, allowing for the Laplace pressure to balance the Kelvin pressure. For the case where synthetic stomata were used, this local increase in humidity is primarily attributed to choking the diffusive vapor flow within the substomatal chambers. For the stomata-free synthetic leaf, the large thickness of the nanoporous ceramic disk allows the water menisci to recede far enough to choke the vapor and self-stabilize while keeping the majority of the nanopores wetted. As a result, we were able to continually transpire water across the stomata-free leaf even at low humidities, avoiding the need for the complex fabrication of stomata. Transpiration rates of the stomata-free leaf varied nonmonotonically with the ambient humidity because of the tradeoff of dry air increasing the retreat length of the menisci. We expect that these findings will facilitate the development of synthetic trees with large throughput capabilities.

\section{RESULTS AND DISCUSSION}

Design of Synthetic Stomata. Our design for vaportrapping synthetic stomata was inspired by mangroves, which are renowned for their highly negative water pressures and excellent stability even in rugged conditions. ${ }^{4,5,45}$ Mangrove saplings (Rhizophora mangle) were obtained from Florida Coastal Mangroves. The received saplings were washed with 


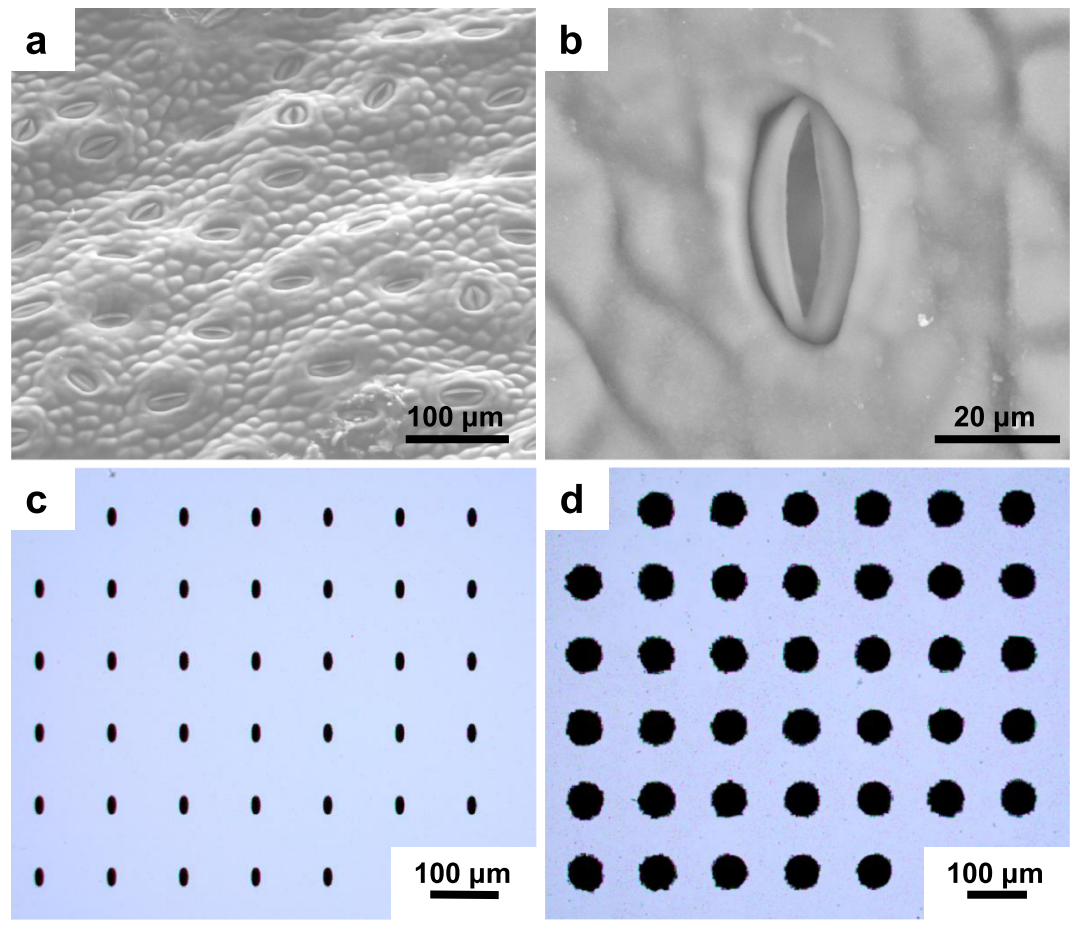

Figure 2. Morphology of natural and synthetic stomata. (a,b) Stomatal apertures on the leaf of a red mangrove plant (Rhizophora mangle). (c) Microfabricated stomatal apertures etched through a silicon wafer, where each aperture is $30 \mu \mathrm{m}$ long, $10 \mu \mathrm{m}$ wide, and has a pitch of $150 \mu \mathrm{m}$ to its neighbors. (d) Substomatal chambers $(60 \mu \mathrm{m}$ diameter, $150 \mu \mathrm{m}$ pitch) etched through a second wafer were aligned directly underneath the apertures. Both silicon wafers were $300 \mu \mathrm{m}$ thick.

water and floated at the free surface of a salt water aquarium by using pieces of foam and twist ties. To obtain the equivalent conditions of the mangroves' natural Floridian habitat, Instant Ocean Sea Salt was used to maintain the salinity and magnesium levels of the water at $21 \mathrm{ppt}$ and $1300 \mathrm{ppm}$, respectively.

Leaf samples were gently plucked from the healthiest mangrove plants. An environmental scanning electron microscope (FEI Quanta 600 FEG) was used to image the topology of the leaves, particularly the topology of the stomata. The stomata were only on the abaxial side of each leaf and the stomatal apertures were observed to be almost exactly $30 \mu \mathrm{m}$ in length with average center-to-center spacings of approximately $150 \mu \mathrm{m}$ between adjacent stomata (Figure 2a). The profile of the stomatal apertures was in the shape of a convex lens with a maximal width of about $10 \mu \mathrm{m}$ (Figure $2 \mathrm{~b}$ ). The topography of the mangrove's substomatal chambers was more difficult to visualize, even when looking at cross sections, but the chambers appeared to exhibit a depth and width of about $60 \mu \mathrm{m}$ each, in reasonable agreement with other reports. ${ }^{46,47}$

Using standard photolithographic techniques, synthetic substomatal chambers and stomatal apertures were microfabricated from silicon wafers. $\mathrm{An} \mathrm{Al}_{2} \mathrm{O}_{3}$ etch mask was patterned on $300 \mu \mathrm{m}$ thick silicon wafers (10.16 cm diameter). Then, either an array of substomatal chambers or an array of stomatal apertures were obtained by Bosch etching all the way through the wafer. Each stomatal aperture exhibited a length of $30 \mu \mathrm{m}$ and a convex width of $10 \mu \mathrm{m}$ to approximate the shape of natural stomata (Figure 2c). This length scale of $\sim 10 \mu \mathrm{m}$ for the apertures is of the same order of magnitude as that reported to optimize the evaporation of water underlying synthetic stomata. ${ }^{48}$ The fixed geometry of our design contrasts with natural stomata, where the apertures can dynamically open and close to tune gas exchange with the environment. ${ }^{49,50}$ A recent work demonstrated hydrogel-based valves that dynamically respond to environmental stimuli, analogous to natural stomata. ${ }^{51}$ Given that our only design consideration is to increase the humidity within the substomatal chambers, it was not necessary for our synthetic stomata to have a dynamic response. For the substomatal chambers, each chamber exhibited a cross-sectional diameter of $60 \mu \mathrm{m}$ and arrays of chambers were organized in a square lattice configuration with the center-to-center pitch between chambers being $150 \mu \mathrm{m}$ (Figure 2d). Alignment marks were placed on each wafer, so that pairs of wafers could be easily oriented to align the substomatal chambers directly beneath the apertures.

Synthetic Floating Leaves. To mimic a natural leaf, the microfabricated stomata were placed atop a nanoporous ceramic disk exhibiting an average pore radius of $80 \mathrm{~nm}$ (see the Materials and Methods section). In our initial setup, a synthetic tree was constructed by placing a microcapillary tube (i.e., "xylem conduit") between the bottom water reservoir and the synthetic leaf (Figure S1). However, the leaf quickly dried out for this tree system (Figure S2), which is readily attributed to the single xylem conduit failing to replenish the water evaporating from the much larger leaf (see calculations in the Supporting Information). To maximize the conductance of water flowing into the leaf, we instead designed a "floating leaf" setup (Figure S3) somewhat analogous to the floating membranes recently used for solar steam generation. ${ }^{52-54}$ This allows the leaf's underside to remain in intimate contact with its water supply even as the reservoir is gradually depleted during transpiration, whereas the leaf's topside is open to the ambient for evaporation. In this manner, we can isolate the effects of humidity on the stability of the water menisci within 

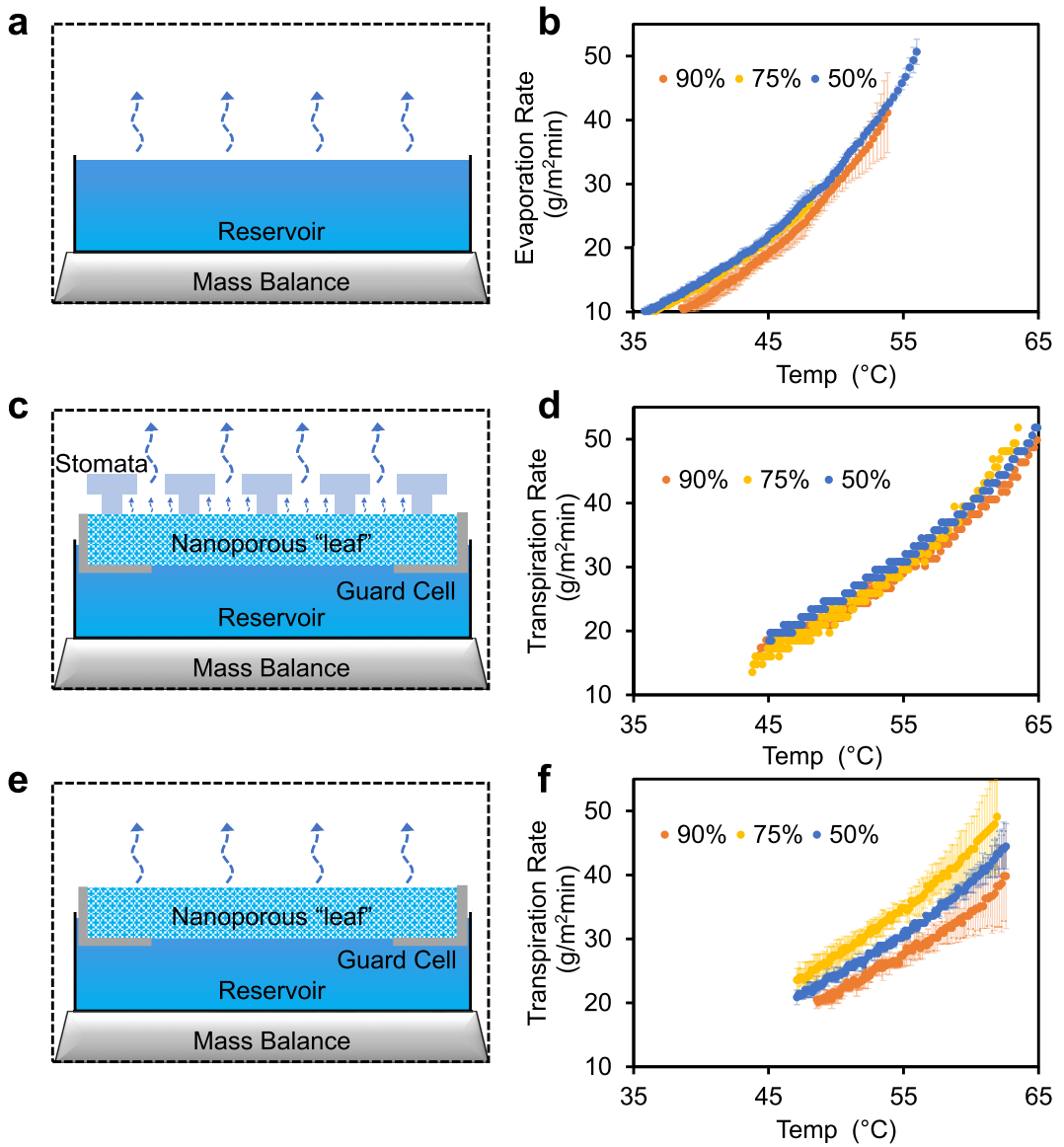

Figure 3. Schematic and results of the three different experimental setups. (a,b) Control case of the evaporation of bulk water from a reservoir; $(\mathrm{c}, \mathrm{d})$ transpiration of water from a synthetic leaf outfitted with stomata floating atop an equivalent reservoir; (e,f) transpiration of water from a floating synthetic leaf that does not have any stomata (nanopores only). Each data series in (b,f) represents an average of three trials with error bars corresponding to a standard deviation. Only three of the five test humidities are shown here for clarity, see Figures S4 and S5 for all the raw data. The water temperatures were somewhat higher when a floating leaf was employed, as the leaf partially insulated the water's heat from the ambient air.

the leaf, without any conflating dry-out events caused by conductance issues.

Three different types of experiments were performed. First, a control experiment was performed that simply measured the evaporation rate of bulk water within the reservoir without adding any synthetic leaf (Figure 3a). A second experiment measured the rate of transpiration across a nanoporous leaf floating atop the reservoir, where the synthetic stomata were placed on the leaf's top face (Figure 3c). Finally, transpiration was measured across a floating nanoporous leaf where the leaf did not include the synthetic stomata (Figure 3e). As the degassed water was still cooling down from the boiling process, the evolving temperature of water within the reservoir was measured by submerging a digital data logger in the water of the reservoir (HOBO Pendant). It was assumed that the menisci within the floating leaf exhibited the same approximate temperature as that measured within the underlying bulk water. By placing the water reservoir on a digital mass balance, the rate of evaporation/transpiration was measured every 1 min over $3 \mathrm{~h}$. The entire setup was placed within a humidity chamber at room temperature with the relative humidity set to either $95,90,85,75$, or $50 \%$.

As expected, for bulk water the averaged evaporation rate increased with decreasing ambient humidity for any given temperature (Figure 3b). While the error bars between adjacent data series do overlap, there is no overlap when comparing the highest and lowest humidities to each other (Figure S4d). The evaporation rate for the bulk water is comparable to the transpiration rate of the floating synthetic leaves, especially at the lower water temperatures. This can be seen indirectly in Figure 3 and is shown explicitly in Figure S5. The comparable evaporation rates are somewhat surprising, considering that the bulk water has a continuous free interface, whereas the nanoporous ceramic has a porosity of only about $32 \%$. This can be partially explained by the curvature of the menisci within the synthetic leaves. However, as will be seen in the analysis below, the negative Laplace pressures and associated curvature are quite modest for our system. Previous reports have found that the evaporation rate of water enclosed within a perforated lid does not necessarily increase as the holes are made larger or more dense. ${ }^{48,55}$ This is because once the water vapor escapes through each pore, it then expands laterally as it diffuses into the ambient. Once these expanding vapor pathways overlap, there is no benefit of further increasing the porosity of the synthetic stomata and the evaporation rate approaches that of bulk water. We expect this similarly explains why the water diffusing from nanopores and/ or stomata in our system is comparable to the evaporation rate of bulk water. 
For the floating synthetic leaf outfitted with stomata, transpiration remained very stable as hypothesized with no apparent dry-out of the leaf (Figure 3d). This excellent stability is most likely due to the vapor emanating from the leaf becoming choked in the substomatal chambers, which increases the local humidity above the water menisci. This choking of humidity within the stomatal chambers also explains why the transpiration rate did not vary significantly with changes in the ambient humidity. In other words, the local concentration gradient above the menisci is mostly regulated by the stomata, with only a weak dependence on the outer humidity. The liquid content was entirely contained within the ceramic disk, whereas only pure water vapor diffused through the synthetic stomata, as the ceramic is porous and superhydrophilic, whereas the silicon-based stomata are only weakly hydrophilic.

For the final experiment, where the vapor-trapping stomata were removed, we expected dry-out would occur because of the much stronger Kelvin pressure acting on the menisci. Even without the stomata the transpiration across the nanoporous leaf displayed remarkable stability over the entire humidity range tested (Figure 3f). Another unusual result obtained by the stomata-free leaf was the nonmonotonic relationship between the transpiration rate and the ambient humidity. For example, the transpiration rate was the largest for $75 \%$ humidity, lowest for $90 \%$, and somewhere in the middle for $50 \%$. This was especially true at the lower range of water temperatures, where the errors bars were smaller and did not tend to overlap between the three different humidities. This is in seeming contradiction with Fick's law, where the evaporation rate should monotonically increase with decreasing humidity. These unexpected findings for the stomata-free nanoporous leaf will now be rationalized by the following model.

Model. The mismatch in water activity between the saturated liquid water $\left(a_{\mathrm{liq}}=1\right)$ in the leaf and the subsaturated water vapor $\left(a_{\text {vap }}=\mathrm{RH}\right)$ in the ambient manifests itself as a pressure governed by the Kelvin equation ${ }^{25}$

$$
P_{\mathrm{K}}=-\frac{R T}{\nu_{\text {liq }}} \ln \left(a_{\text {vap }}\right)
$$

where $P_{\mathrm{K}}$ is the Kelvin pressure acting on the menisci, $R$ is the universal gas constant, $T$ is the temperature at the menisci's interface, $\nu_{\text {liq }}$ is the molar volume of liquid water, and $a_{\text {vap }}=$ $P_{\text {vap }} / P_{\text {sat }}$ is the activity of the water vapor directly above the meniscus. For the five ambient humidities tested, $\mathrm{RH}=95,90$, 85,75 , and $50 \%$, the initial Kelvin pressures acting on the menisci are $P_{\mathrm{K}}=7.06,14.5,22.4,39.6$, and $95.4 \mathrm{MPa}$, respectively, under room temperature conditions.

To maintain equilibrium, the Kelvin pressure $P_{\mathrm{K}}$ needs to be matched by a negative Laplace pressure of the same magnitude. Such a negative Laplace pressure can be obtained by the water menisci assuming a concave curvature, as given by the Laplace equation

$$
P_{\mathrm{L}}=-\frac{2 \sigma \cos \theta}{r_{\text {pore }}}
$$

where $\sigma$ is the surface tension of water, $\theta$ is the contact angle of each meniscus along the side walls of the pores, and $r_{\text {pore }}$ is the average radius of the pores in the leaf. Neglecting contact angle hysteresis effects, such that $\cos \theta \approx 1$, results in a maximum possible Laplace pressure of $P_{\mathrm{L}, \max }=-1.82 \mathrm{MPa}$ for our pore size of $r_{\text {pore }} \approx 80 \mathrm{~nm}$. This Laplace pressure is much smaller than all of the Kelvin pressures calculated for all five ambient humidities. Indeed, for our nanopores a humidity of about $99 \%$ would have been required for even the maximal Laplace pressure to balance the Kelvin pressure. For our test humidities, it was therefore impossible for the menisci to achieve equilibrium, such that they become unstable and begin to recede from the top of the nanoporous leaf. This is what motivated the fabrication of the synthetic stomata, which can increase the local humidity above the menisci to decrease $P_{\mathrm{K}}$ such that it can now be matched by $P_{\mathrm{L}}$. The excellent stability of the water even within our stomata-free synthetic leaf reveals that nanopores alone are sufficient for increasing the local humidity to an equilibrium value.

Besides the requirement of balancing the Kelvin and Laplace pressures at steady state, a second requirement not often discussed is the conservation of mass of transpiration. Liquid water is highly incompressible, which mandates that the flow rate of liquid being pumped across the leaf cannot exceed the evaporation rate from the leaf's menisci. In the absence of xylem conduits, the volumetric flow rate $Q$ across the synthetic leaf system is simply given by Darcy's Law

$$
\Delta P_{\text {leaf }}=\frac{Q t}{\kappa A}
$$

where $\Delta P_{\text {leaf }}=\left|P_{\mathrm{L}}\right|$ is the pressure drop across the leaf, $t=7$ $\mathrm{mm}$ is the thickness of the porous disk, $A$ is the cross-sectional area of the floating leaf (with a diameter of $105 \mathrm{~mm}$ ), and $\kappa=$ $\left(\varphi r_{\text {pore }}^{2}\right) /(8 \mu \tau)$ is the hydraulic permeability of the nanopores with respect to liquid water, where $\varphi$ is the porosity, $\tau$ is the tortuosity, and $\mu$ is the water's viscosity. Using the commercially available values of $\varphi \approx 0.32$ and $r_{\text {pore }} \approx 80 \mathrm{~nm}$, holding the viscosity constant at $\mu \approx 0.89 \mathrm{mPa} \cdot \mathrm{s}$ (i.e., neglecting minor variations with water temperature), and estimating the tortuosity as $\tau \approx 3.5$, yields a hydraulic permeability of $\kappa \approx 8.2 \times 10^{-14} \mathrm{~m}^{2} / \mathrm{Pa} \cdot \mathrm{s}$. The choice of $\tau \approx 3.5$ is slightly higher than the value of $\tau \approx 3$ corresponding to an ideal isotropic architecture of cylindrical pores, in order to account for the meandering nature of the pore network. ${ }^{56,57}$

For ambient relative humidities of $95,90,85,75$, and $50 \%$, the respective flow rates measured at a water temperature of 50 ${ }^{\circ} \mathrm{C}$ were $Q=3.3 \pm 0.6,3.06 \pm 0.19,3.39 \pm 0.10,3.8 \pm 0.2$, and $3.44 \pm 0.10 \mathrm{~mm}^{3} / \mathrm{s}$ for the stomata-free leaf. Values of $Q$ were obtained by measuring the mass flow rate of transpiration $(\dot{m})$ with the mass balance, where $Q=\dot{m} / \rho$. Plugging these values of $Q$ into eq 3 yields estimated pressure drops of $\Delta P_{\text {leaf }}=33 \pm$ $6,30.2 \pm 1.9,33.5 \pm 1.0,37 \pm 2$, and $34.0 \pm 1.0 \mathrm{kPa}$, respectively, across the synthetic leaf. These Laplace pressures are 2 orders of magnitude less than the maximum possible Laplace pressure calculated from eq 2 . This indicates that in our case it is actually the diffusive evaporation rate and the nanopore geometry, as opposed to the Kelvin pressure, that prescribe the appropriate Laplace pressure such that mass is conserved during transpiration. Of course, the Kelvin pressure (eq 1) still needs to be matched to this weak Laplace/Darcy pressure, such that the local humidity above the menisci must be nearly $100 \%$ at equilibrium.

For water menisci exposed to subsaturated air, the critical retreat distance $l$ of the menisci is given by ${ }^{34}$

$$
\frac{l}{t-l}=\frac{P_{c}-P_{\text {ext }}}{-P_{c}} \times \beta
$$


a

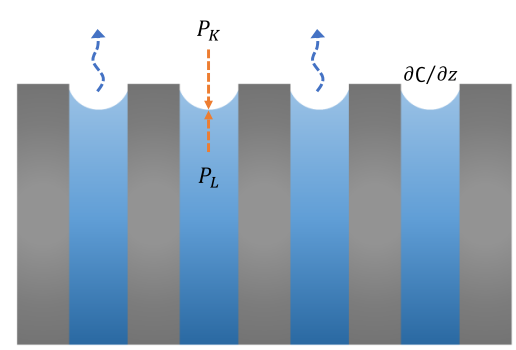

C

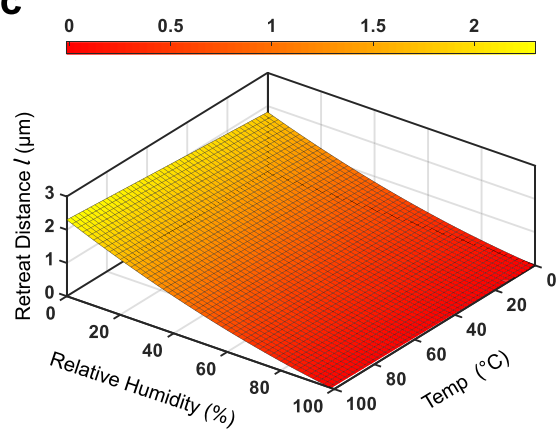

b

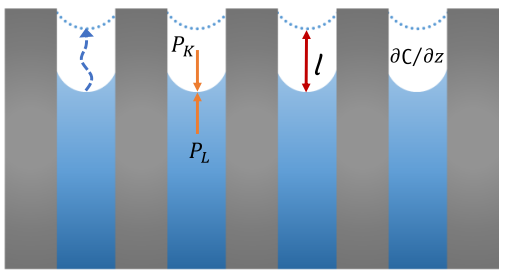

d

$800 \quad 900 \quad 1000 \quad 1100 \quad 1200 \quad 1300 \quad 1400 \quad 1500$

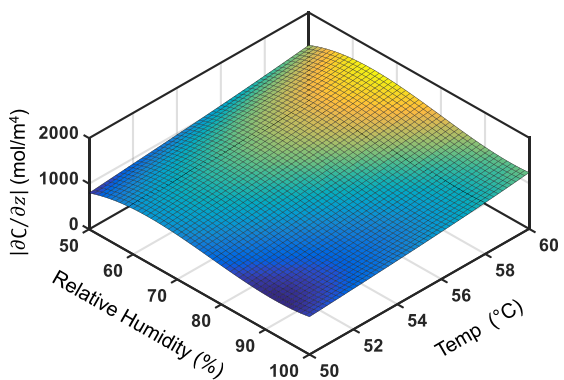

Figure 4. Model of retreat distances and boundary layer gradients for meniscus stabilization. (a) For stomata-free synthetic leaves, the menisci were initially near the outer surface of the leaf and unstable $\left(P_{\mathrm{K}}>P_{\mathrm{L}}\right)$. (b) Unbalanced menisci retreat within the pores, which increases the local humidity until reaching a distance $l$ where equilibrium is established $\left(P_{\mathrm{K}}=P_{\mathrm{L}}\right)$. (c) Retreat distance required for equilibrium, graphed as a function of meniscus temperature and the ambient humidity (eq 4). (d) Experimental measurements of the average transpiration rate of stomata-free leaves were used to estimate the boundary layer concentration gradient (eq 5 ).

where the disk thickness $t$ represents the permeation pathway, $P_{c}$ is the capillary pressure corresponding to an intrinsic contact angle of $\cos \theta \approx 1\left(P_{\mathrm{L}, \max }\right), P_{\text {ext }}$ is the Kelvin pressure corresponding to the ambient humidity using eq 1 , and $\beta$ is the ratio of conductances. Specifically, $\beta=g_{\text {vap }} / g_{\text {liqu }}$, where $g_{\text {liq }}$ is the linear mass conductivity of a liquid-filled pore and $g_{\text {vap }}$ the linear mass conductivity of a vapor-filled pore. For our pore size of $r_{\text {pore }}=80 \mathrm{~nm}, \beta \approx 2.1 \times 10^{-6}$. Conceptually, eq 4 can be thought of as expressing the initial mismatch between the Kelvin and Laplace pressures to determine the extent of local humidification (i.e., recession) required to stabilize the menisci (Figure 4a,b). In Figure 4c, values of $l$ are calculated for any possible meniscus temperature and initial humidity. The retreat distance varies from $l \approx 0$ to $2 \mu \mathrm{m}$ depending on the humidity, while also increasing weakly with increasing temperature. Note that for our case of evaporation-limited flow, a retreat distance slightly larger than that predicted by eq 4 is required as the Laplace pressure is much lesser than its maximal value. These findings indicate that even our relatively large pore size can facilitate stable transpiration over a wide humidity range, provided that the leaf thickness is at least micrometric. This retraction of the air-water menisci may not be directly applicable to natural plants, whose cellular wall matrix is a porous material infiltrated by a gel-like substance, ${ }^{58,59}$ where the gel is swollen by water in a manner described by the Flory-Rehner equation. ${ }^{25,60,61}$ However, the stomata of natural plants serve to increase the local humidity, as evidenced by our results with leaves containing synthetic stomata. Therefore, this retreating meniscus model is only necessitated for the specific case of rigid, stomata-free synthetic leaves.

The air in our experiments was room temperature without any radiative heat source or air currents, such that the evaporation was diffusive in nature. According to Fick's first law, the diffusive evaporation rate is governed by

$$
J=-D_{\mathrm{f}} \frac{\partial C}{\partial z}
$$

where $J$ is the diffusive flux, $D_{\mathrm{f}} \approx 2.82 \times 10^{-5} \mathrm{~m}^{2} / \mathrm{s}$ is the diffusion coefficient of water vapor in air, and $\partial \mathrm{C} / \partial \mathrm{z}$ is the local gradient in water vapor concentration directly above each meniscus. By plugging in the empirical measurements of $J$ shown in Figure 3f, the concentration gradient $\partial C / \partial z$ can be plotted against the tested ambient humidities and water temperatures. A polynomial equation was then used to obtain a smooth fit between these discrete points, resulting in the continuous curve for $\partial C / \partial z$ shown in Figure $4 d$. For any given water temperature, this curve shows that the concentration gradient varies nonmonotonically with the ambient humidity, with the largest gradient corresponding to about $70 \%$ humidity.

The nonmonotonic dependence of the transpiration rate on the ambient humidity can be conceptualized as a dual constraint problem. Low ambient humidities exhibit high degrees of subsaturation, increasing the $\partial C / \partial z$ value, but also force menisci to retreat by a larger distance $l$ into the nanopores, which decreases $\partial C / \partial z$ because of the local humidification. On the other extreme, high ambient humidities are only weakly subsaturated, but also minimize $l$ which largely prevents humidification within the pores. Thus, both extremes result in a decrease in the local concentration gradient above the meniscus, with an intermediate humidity value providing the best compromise between the constraints. This dual constraint hypothesis is a plausible explanation for why water evaporates at the same rate within the leaf for 95 and $50 \%$ humidities (Figure S4), whereas a middle-ground humidity (75\%) exhibited the largest evaporation rate. We encourage future works to employ numerical methods to test this dual constraint hypothesis for evaporation occurring within a variety of nanopore (and stomata) geometries. It is also possible that 
some of these findings would be relevant beyond our scope of natural and synthetic plants, for example, water evaporating from soil ${ }^{62}$ or permeable rock. ${ }^{63}$

\section{CONCLUSIONS}

Using nanoporous synthetic leaves, we demonstrated that transpiration is self-stabilizing over a wide ambient humidity range both with and without stomata. For leaves overlaid with stomata, the evaporative vapor flow becomes choked within the stomatal chambers. For stomata-free leaves, the menisci partially recede into the leaf to choke the vapor within the nanopores themselves. In both cases, the resulting local increase in humidity serves to decrease the Kelvin pressure acting on the concave menisci, such that it can be balanced by the negative Laplace pressure. To conserve mass, the magnitude of the negative Laplace pressure driving Darcy flow across the leaf is prescribed by the leaf's evaporation rate. Leaves with stomata exhibited a remarkably consistent evaporation rate over a range of ambient humidities, which was attributed to the stomata regulating the diffusive flux of vapor from the menisci. For the stomata-free leaves, the selfstabilizing recession of the menisci resulted in a nonmonotonic dependence of the transpiration rate on the ambient humidity. Low ambient humidities maximized the difference in vapor concentration (to enhance evaporation), but also increased the menisci retreat length (diminishing evaporation), and vice versa for high ambient humidities. These findings indicate that incorporating stomata into synthetic leaves is attractive when wanting to minimize variations in the transpiration rate, whereas stomata-free leaves are more cost-effective to fabricate and can still avoid dry-out when sufficiently thick.

\section{EXPERIMENTAL SECTION}

Materials and Methods. To mimic the leaf tissue, porous ceramic disks were bought from Soilmoisture Equipment Corp. Each ceramic disk was $105 \mathrm{~mm}$ in diameter, $7 \mathrm{~mm}$ thick, had an average pore size of $r_{\text {pore }}=80 \mathrm{~nm}$, and a porosity of $32 \%$ by volume. This ceramic disk was held within a polycarbonate disk featuring a circular hole, $8.9 \mathrm{~cm}$ in diameter, in the floor of the recess to allow for the intake of water into the bottom face of the ceramic disk. A reservoir of degassed water was held within a hollowed out cylindrical block of polyvinyl chloride (PVC) (McMaster Carr). Floating was enabled by securing a ring of polyethylene foam around the outer rim of the polycarbonate disk.

Experimental Setup. To fill the nanoporous synthetic leaf with degassed water, it was vigorously boiled in a pot of water for $1 \mathrm{~h}$. Prior to placing the leaf in the boiling water, the water was itself first degassed by boiling for about $3 \mathrm{~h}$. After boiling, the ceramic disk was seated within a recess cut within a polycarbonate disk. The polycarbonate disk and enclosed synthetic leaf were then lifted out of the pot of water and floated at the free interface of a reservoir. The foam was designed to nearly hug the inner walls of the PVC reservoir, such that the vast majority of the water evaporated from the wetted leaf and not from the bulk water itself. The seam between the rim of the ceramic disk and the side wall of the polycarbonate recess was sealed with Parafilm, to ensure that evaporation was only occurring along the top face of the ceramic leaf. For select experiments, the synthetic stomatal layers were placed atop the ceramic leaf prior to sealing with the Parafilm. For the control case of bulk water only, the PVC reservoir was simply filled with degassed water but with no leaf on top. As the degassed water was still warm from the boiling process, the temperature of water within the reservoir was measured by submerging a digital data logger in the water of the reservoir (HOBO Pendant). The ambient humidity of the surrounding air was set using a large humidity chamber (Electro-Tech Systems, Inc.) that enclosed the setup (see Figure S3). A digital mass balance was used to measure the transpiration rate of water across the synthetic leaf, with three trials performed for each ambient humidity.

\section{ASSOCIATED CONTENT}

Supporting Information

The Supporting Information is available free of charge on the ACS Publications website at DOI: 10.1021/acsami.9b00041.

Single tree setup with single xylem tube; flow rate up the synthetic tree; photo of floating synthetic leaf setup; evaporation/transpiration rates for all five humidities; and comparing evaporation/transpiration rates for each setup (PDF)

\section{AUTHOR INFORMATION}

\section{Corresponding Author}

*E-mail: boreyko@vt.edu.

\section{Author Contributions}

W.S., J.R.V., B.R.S., C.P.C., A.S.B., and J.B.B. designed the research. W.S., J.R.V., M.W.R., and D.A.S. collected the data. W.S. and J.B.B. analyzed the data. W.S. and J.B.B. wrote the paper.

\section{Notes}

The authors declare no competing financial interest.

\section{ACKNOWLEDGMENTS}

This work was supported by a National Science Foundation CAREER Award (CBET-1653631) and by the Ralph E. Powe Junior Faculty Enhancement Award (ORAU-459204). The microfabrication of the stomata was conducted at the Center for Nanophase Materials Sciences (CNMS) at the Oak Ridge National Laboratory as a user project (User Project CNMS2015-R48). The illustration of the tree shown in Figure la was drawn by Mary Katherine Wooten. We thank Andrew Fugaro for technical assistance in making the three-dimensional plots.

\section{REFERENCES}

(1) Koch, G. W.; Sillett, S. C.; Jennings, G. M.; Davis, S. D. The limits to tree height. Nature 2004, 428, 851-854.

(2) Holbrook, N. M.; Zwieniecki, M. A. Vascular Transport in Plants; Academic Press, 2011.

(3) Stroock, A. D.; Pagay, V. V.; Zwieniecki, M. A.; Holbrook, N. M. The physicochemical hydrodynamics of vascular plants. Annu. Rev. Fluid. Mech. 2014, 46, 615-642.

(4) Scholander, P. F. How mangroves desalinate seawater. Physiol. Plant. 1968, 21, 251-261.

(5) Parida, A. K.; Jha, B. Salt tolerance mechanisms in mangroves: a review. Trees 2010, 24, 199-217.

(6) Kim, K.; Seo, E.; Chang, S. K.; Park, T. J.; Lee, S. J. Novel water filtration of saline water in the outermost layer of mangrove roots. Sci. Rep. 2016, 6, 20426.

(7) Paudel, I.; Naor, A.; Gal, Y.; Cohen, S. Simulating nectarine tree transpiration and dynamic water storage from responses of leaf conductance to light and sap flow to stem water potential and vapor pressure deficit. Tree Physiol. 2015, 35, 425-438.

(8) Ayyoub, A.; Er-Raki, S.; Khabba, S.; Merlin, O.; Ezzahar, J.; Rodriguez, J. C.; Bahlaoui, A.; Chehbouni, A. A simple and alternative approach based on reference evapotranspiration and leaf area index for estimating tree transpiration in semi-arid regions. Agric. Water Manag. 2017, 188, 61-68.

(9) Novick, K. A.; Miniat, C. F.; Vose, J. M. Drought limitations to leaf-level gas exchange: results from a model linking stomatal optimization and cohesion-tension theory. Plant Cell Environ. 2016, 39, 583-596. 
(10) Schenk, H. J.; Espino, S.; Romo, D. M.; Nima, N.; Do, A. Y. T.; Michaud, J. M.; Papahadjopoulos-Sternberg, B.; Yang, J.; Zuo, Y. Y.; Steppe, K.; Jansen, S. Xylem surfactants introduce a new element to the cohesion-tension theory. Plant Physiol. 2016, 173, 1177.

(11) Bentrup, F.-W. Water ascent in trees and lianas: the cohesiontension theory revisited in the wake of Otto Renner. Protoplasma 2017, 254, 627-633.

(12) Nardini, A.; Lo Gullo, M. A.; Salleo, S. Refilling embolized xylem conduits: Is it a matter of phloem unloading? Plant Sci. 2011, 180, 604-611.

(13) Lens, F.; Tixier, A.; Cochard, H.; Sperry, J. S.; Jansen, S.; Herbette, S. Embolism resistance as a key mechanism to understand adaptive plant strategies. Curr. Opin. Plant Biol. 2013, 16, 287-292.

(14) Caupin, F.; Arvengas, A.; Davitt, K.; Azouzi, M. E. M.; Shmulovich, K. I.; Ramboz, C.; Sessoms, D. A.; Stroock, A. D. Exploring water and other liquids at negative pressure. J. Phys.: Condens. Matter 2012, 24, 284110.

(15) Brown, H. R. The theory of the rise of sap in trees: some historical and conceptual remarks. Phys. Perspect. 2013, 15, 320-358.

(16) Cai, J.; Tyree, M. T. Measuring vessel length in vascular plants: can we divine the truth? History, theory, methods, and contrasting models. Trees 2014, 28, 643-655.

(17) Petit, G.; Crivellaro, A. Comparative axial widening of phloem and xylem conduits in small woody plants. Trees 2014, 28, 915-921.

(18) Kim, H. K.; Park, J.; Hwang, I. Investigating water transport through the xylem network in vascular plants. J. Exp. Bot. 2014, 65, 1895-1904.

(19) Tyree, M. T.; Zimmermann, M. H. Xylem Structure and the Ascent of Sap; Springer Science \& Business Media, 2013.

(20) Wheeler, T. D.; Stroock, A. D. Stability Limit of Liquid Water in Metastable Equilibrium with Subsaturated Vapors. Langmuir 2009, $25,7609-7622$.

(21) Chen, I.-T.; Sessoms, D. A.; Sherman, Z.; Choi, E.; Vincent, O.; Stroock, A. D. Stability limit of water by metastable vapor-liquid equilibrium with nanoporous silicon membranes. J. Phys. Chem. B 2016, 120, 5209-5222.

(22) Zimmermann, U.; Schneider, H.; Wegner, L. H.; Haase, A. Water ascent in tall trees: does evolution of land plants rely on a highly metastable state? New Phytol. 2004, 162, 575-615.

(23) García-Valladares, O.; Pérez-Segarra, C. D.; Oliva, A. Numerical simulation of capillary-tube expansion devices behaviour with pure and mixed refrigerants considering metastable region. Part II: experimental validation and parametric studies. Appl. Therm. Eng. 2002, 22, 379-391.

(24) Dixon, H. H.; Joly, J. On the ascent of sap. Philos. Trans. R. Soc., B 1895, 186, 563-576.

(25) Wheeler, T. D.; Stroock, A. D. The transpiration of water at negative pressures in a synthetic tree. Nature 2008, 455, 208-212.

(26) Li, J.-m.; Liu, C.; Xu, Z.; Zhang, K.-p.; Ke, X.; Li, C.-y.; Wang, L.-d. A bio-inspired micropump based on stomatal transpiration in plants. Lab Chip 2011, 11, 2785-2789.

(27) Jingmin, L.; Chong, L.; Zheng, X.; Kaiping, Z.; Xue, K.; Liding, W. A microfluidic pump/valve inspired by xylem embolism and transpiration in plants. PLoS One 2012, 7, No. e50320.

(28) Lamb, M.; Koch, G. W.; Morgan, E. R.; Shafer, M. W. A synthetic leaf: the biomimetic potential of graphene oxide. Proceedings of SPIE, 2015; Vol. 9429, pp 1-10.

(29) Zhu, M.; Li, Y.; Chen, G.; Jiang, F.; Yang, Z.; Luo, X.; Wang, Y.; Lacey, S. D.; Dai, J.; Wang, C.; Jia, C.; Wan, J.; Yao, Y.; Gong, A.; Yang, B.; Yu, Z.; Das, S.; Hu, L. Tree-inspired design for highefficiency water extraction. Adv. Mater. 2017, 29, 1704107.

(30) Vincent, O.; Marmottant, P.; Quinto-Su, P. A.; Ohl, C. D. Birth and growth of cavitation bubbles within water under tension confined in a simple synthetic tree. Phys. Rev. Lett. 2012, 108, 184502.

(31) Duan, C.; Karnik, R.; Lu, M.-C.; Majumdar, A. Evaporationinduced cavitation in nanofluidic channels. Proc. Natl. Acad. Sci. U.S.A. 2012, 109, 3688-3693.

(32) Vincent, O.; Sessoms, D. A.; Huber, E. J.; Guioth, J.; Stroock, A. D. Drying by cavitation and poroelastic relaxations in porous media with macroscopic pores connected by nanoscale throats. Phys. Rev. Lett. 2014, 113, 134501.

(33) Noblin, X.; Mahadevan, L.; Coomaraswamy, I. A.; Weitz, D. A.; Holbrook, N. M.; Zwieniecki, M. A. Optimal vein density in artificial and real leaves. Proc. Natl. Acad. Sci. U. S. A. 2008, 105, 9140-9144.

(34) Vincent, O.; Szenicer, A.; Stroock, A. D. Capillarity-driven flows at the continuum limit. Soft Matter 2016, 12, 6656-6661.

(35) Kim, H.; Kim, K.; Lee, S. Compact and thermosensitive natureinspired micropump. Sci. Rep. 2016, 6, 36085.

(36) Borno, R. T.; Steinmeyer, J. D.; Maharbiz, M. M. Chargepumping in a synthetic leaf for harvesting energy from evaporationdriven flows. Appl. Phys. Lett. 2009, 95, 013705.

(37) Gruber, P.; Rupp, A. I. K. S. Investigation of leaf shape and edge design for faster evaporation in biomimetic heat dissipation systems. Bioinspiration, Biomimetics, and Bioreplication VIII, 2018; Vol. 10593, p $105930 \mathrm{H}$.

(38) Chen, I.-T.; Pharkya, A.; Stroock, A. D. Analysis of superheated loop heat pipes exploiting nanoporous wick membranes. AIChE J. 2014, 60, 762-777.

(39) Pagay, V.; Santiago, M.; Sessoms, D. A.; Huber, E. J.; Vincent, O.; Pharkya, A.; Corso, T. N.; Lakso, A. N.; Stroock, A. D. A microtensiometer capable of measuring water potentials below -10 MPa. Lab Chip 2014, 14, 2806-2817.

(40) Stroock, A. D.; Lakso, A. N.; Pagay, V.; Santiago, M.; Sessoms, D. Mems Microtensiometer. U.S. Patent Application No. 15/689,584; 2018.

(41) Riederer, M.; Schreiber, L. Protecting against water loss: analysis of the barrier properties of plant cuticles. J. Exp. Bot. 2001, 52, 2023-2032.

(42) Fites, J. A.; Teskey, R. O. $\mathrm{CO}_{2}$ and water vapor exchange of Pinustaeda in relation to stomatal behavior: test of an optimization hypothesis. Can. J. For. Res. 1988, 18, 150-157.

(43) Guehl, J.-M.; Aussenac, G. Photosynthesis Decrease and Stomatal Control of Gas Exchange in Abies alba Mill. in Response to Vapor Pressure Difference. Plant Physiol. 1987, 83, 316-322.

(44) Meinzer, F. C. The effect of vapor pressure on stomatal control of gas exchange in Douglas fir (Pseudotsuga menziesii) saplings. Oecologia 1982, 54, 236-242.

(45) Popp, M.; Polania, J.; Weiper, M. Physiological adaptations to different salinity levels in mangrove. Towards the Rational use of High Salinity Tolerant Plants; Springer, 1993; Vol. 27, pp 217-224.

(46) Pickard, W. F. How does the shape of the substomatal chamber affect transpirational water loss? Math. Biosci. 1981, 56, 111-127.

(47) Roth-Nebelsick, A. Computer-based studies of diffusion through stomata of different architecture. Ann. Bot. 2007, 100, 23-32.

(48) Zwieniecki, M. A.; Haaning, K. S.; Boyce, C. K.; Jensen, K. H. Stomatal design principles in synthetic and real leaves. J. R. Soc. Interface 2016, 13, 20160535.

(49) Berg, H. C. Random Walks in Biology; Princeton University Press, 1993.

(50) Kim, H.; Lee, S. J. Stomata-inspired membrane produced through photopolymerization patterning. Adv. Funct. Mater. 2015, 25, 4496-4505.

(51) Gargava, A.; Arya, C.; Raghavan, S. R. Smart hydrogel-based valves inspired by the stomata in plants. ACS Appl. Mater. Interfaces 2016, 8, 18430-18438.

(52) Ghasemi, H.; Ni, G.; Marconnet, A. M.; Loomis, J.; Yerci, S.; Miljkovic, N.; Chen, G. Solar steam generation by heat localization. Nat. Commun. 2014, 5, 4449.

(53) Ito, Y.; Tanabe, Y.; Han, J.; Fujita, T.; Tanigaki, K.; Chen, M. Multifunctional porous graphene for high-efficiency steam generation by heat localization. Adv. Mater. 2015, 27, 4302-4307.

(54) Li, T.; Liu, H.; Zhao, X.; Chen, G.; Dai, J.; Pastel, G.; Jia, C.; Chen, C.; Hitz, E.; Siddhartha, D.; Yang, R.; Hu, L. Scalable and highly efficient mesoporous wood-based solar steam generation device: Localized heat, rapid water transport. Adv. Funct. Mater. 2018, 28, 1707134

(55) Ting, I. P.; Loomis, W. E. Diffusion through stomates. Am. J. Bot. 1963, 50, 866-872. 
(56) Gruener, S.; Wallacher, D.; Greulich, S.; Busch, M.; Huber, P. Hydraulic transport across hydrophilic and hydrophobic nanopores: Flow experiments with water and n-hexane. Phys. Rev. E 2016, 93, 013102.

(57) Crossley, P. A.; Schwartz, L. M.; Banavar, J. R. Image-based models of porous media: Application to Vycor glass and carbonate rocks. Appl. Phys. Lett. 1991, 59, 3553-3555.

(58) Pickard, W. F. The ascent of sap in plants. Prog. Biophys. Mol. Biol. 1981, 37, 181-229.

(59) Rand, R. H. Fluid mechanics of green plants. Annu. Rev. Fluid. Mech. 1983, 15, 29-45.

(60) Hong, W.; Zhao, X.; Zhou, J.; Suo, Z. A theory of coupled diffusion and large deformation in polymeric gels. J. Mech. Phys. Solids 2008, 56, 1779-1793.

(61) Hong, W.; Zhao, X.; Suo, Z. Large deformation and electrochemistry of polyelectrolyte gels. J. Mech. Phys. Solids 2010, $58,558-577$.

(62) Bittelli, M.; Ventura, F.; Campbell, G. S.; Snyder, R. L.; Gallegati, F.; Pisa, P. R. Coupling of heat, water vapor, and liquid water fluxes to compute evaporation in bare soils. J. Hydrol. 2008, $362,191-205$.

(63) Tsypkin, G. G.; Woods, A. W. Vapour extraction from a watersaturated geothermal reservoir. J. Fluid Mech. 2004, 506, 315-330. 